4. Using the bounds on $x$ thus derived, the curve for a given value of $\gamma$ is calculated and plotted.

5. The value of $\gamma$ is incremented by a specified integral number of degrees and step 3 repeated until $\gamma$ does not satisfy the inequality (5).

Some of the curves are marked with the corresponding values of $\gamma$, and the $x$ axis and origin are marked. The diameter of the chart is limited to a maximum of 10 inches by the graph plotter dimensions.

The program takes approximately 1.5 minutes per chart to prepare a magnetic tape and the standard program plotting the chart takes about 6 minutes. It is worth while to note that hand tracing a chart, starting from the computed points, requires a full working day.

The plotted chart is photographed with a graduate circle of $360^{\circ}$; the negative of this is used to produce a chart of radius $R$ on a transparent positive film.

The Fig. 1 shows a chart of radius $R=35 \mathrm{~mm}$ and a working distance $t=65.9 \mathrm{~mm}$. This chart is used for measuring the two angular parameters defining the orientation of each diffraction line $\{111\}$ present on the film, obtained with the characteristic radiation $L \alpha_{1}$ of tin, with an aluminum specimen.

The authors would like to acknowledge the assistance of Dr R. Doherty in the preparation of this note, and the S.R.C. for financial assistance.

\section{References}

Bevis, M. \& Swindells, N. (1967). Phys. Stat. Sol. 20, 197. HÄLBIG, H., RYdER, P. L. \& PITSCH, W. (1968). V International Congress on $X$-ray Optics and Microanalysis, p. 388. Berlin: Springer Verlag.

MACKAY, K. J. H. (1965). IV International Congress on $X$ ray Optics and Microanalysis, p. 544. Paris: Hermann.

Morris, W. G. (1966). General Electric Report No. 66-C-217.

Peters, E. T. \& Ogilvie, R. E. (1965). Trans. Met. Soc. $A I M E, 233,89$.

Rowland, P. \& Bevis, M. (1968). Phys. Stat. Sol. 26, K 25.

\title{
Reference Information
}

\author{
J. Appl. Cryst. (1970). 3, 421
}

Generation of $\bar{\alpha}$-SiC stacking sequences. * By R. B. Roof, JR † and R. L. Bivins, University of California, Los Alamos Scientific Laboratory, Los Alamos, New Mexico 87544, U.S.A.

(Received 23 December 1969)

\begin{abstract}
$\alpha-\mathrm{SiC}$ crystal structures are polymorphs usually consisting of 2-, 3- and 4-layer blocks arranged in stacking sequences in order to yield a specific number of layers in a hexagonal unit cell. A computer-generated tabulation of some combinations of these blocks into significant sequences representing possible crystal structures is available from the authors.
\end{abstract}

$\alpha$-SiC displays a large number of polytypes which are associated with long-range ordering of stacking sequences. The various structures may be described by a sequence of numbers designating the right and left, or 'zigzag', addition of layers to an initial layer. Most $\alpha$-SiC polytypes are built by the combination of blocks consisting of 2,3 , and 4 layers, are described in the hexagonal lattice system, and exist in two varieties, hexagonal and rhombohedral.

A computer-generated tabulation has been prepared of possible zigzag sequences to build to a given number of layers. To qualify for inclusion in the tabulation, the zigzag sequence was required to display the following characteristics: (i) It must contain an even number of digits. (ii) The sum of the digits having alternating algebraic signs must be evenly divisible by 3 . (iii) It must not contain multiple cells of a lower layer number.

* Work performed under the auspices of the U.S. Atomic Energy Commission.

+ Present address: Department of Physics, University of Western Australia, Nedlands 6009, Australia.
In a report available on request from the present authors (Los Alamos Scientific Laboratory Report LA-4295) there are listed in tabular form the sequences for a given layer number for the following cases: $(a)$ sequences containing the digits 2,3 , and 4 for layer numbers 2 through $30,(b)$ sequences containing the digits 2,3 for layer numbers 10 through 45 , and $(c)$ sequences containing the digits 3,4 for layer numbers 10 through 64 .

We note that of the $76 \alpha$-SiC polytypes identified (Shaffer, 1969; Gomes de Mesquita, 1965, 1968), 34 have been structurally determined. Of these, 30 are composed exclusively of sequences containing just the digits 2,3 or 3,4 . Since it appears that the large majority of polymorphs as yet structurally undetermined might reasonably be represented by sequence of these types, we included cases (b) and (c) in the report.

\section{References}

Gomes de Mesquita, A. H. (1965). Acta Cryst. 18, 128. Gomes de Mesquita, A. H. (1968). Acta Cryst. B 24, 1461. Shaffer, P. T. B. (1969). Acta Cryst. B 25, 477. 